\title{
ANALISIS DEL COMPORTAMIENTO DE LOS RESIDUOS DE BOTELLAS DE VIDRIO DE BEBIDAS ALCOHÓLICAS. ESTUDIO DE CASO MUNICIPIO DE NEIVA
}

\section{LIQUEUR BOTTLE GLASS WASTE BEHAVIOUR ANALISYS. CASE STUDY IN THE NEIVA CITY}

\section{Christian Felipe Valderrama Lopez}

Ingeniero Ambiental y Sanitario, Magister Sistemas integrales de gestión y prevención de riesgos laborales

Universidad Nacional Abierta y a Distancia

christian.valderrama@unad.edu.co

\section{RESUMEN.}

Los residuos sólidos se han convertido en una gran problemática a nivel global debido a que la gran mayoría de estos no están siendo recuperados, terminando en rellenos sanitarios, botaderos a cielo abierto o en otras actividades ilegales. Un caso particular es el de las botellas recipientes de alcohol fabricadas en vidrio, las cuales tienen muy poco valor dentro de la economía tradicional del reciclaje pero un alto precio dentro del mercado del alcohol adulterado, es por ello que este proyecto de investigación realizado en la ciduad de Neiva en el año 2017, determinólos parámetros de manejo de los residuos de botellas de vidrio en los establecimientos nocturnos dentro del casco urbano, con el fin de identificar el comportamiento del residuo y poder proponer alternativas viables que permitirán ser reincorporados dentro del ciclo de vida. Para su desarrollo se estableciócomo indicador las botellas de bebidas alcohólicas mayor a 20 grados alcolimetricos, se trabajo en las comunas $3,4,6$, debido a que la mayor población de establecimientos se encuentran allí, se utilizó el método de observación directa en cada establecimiento y se realizaron entrevistas a entidades recicladoras y cooperativas de recuperación de los residuos. Se encontró un poco de gestión ambiental por parte de los establecimientos nocturnos, un mercado negro que surge a partir de algunos recuperadores informales y un producto como el vidrio que no se considera viable económicamente dentro del proceso de recuperación local.

DOI: https://doi.org/10.22490/ECAPMA.3418 
Palabras Clave: Comportamiento, Manejo, Botellas, Vidrio, Establecimientos, Reciladoras.

\section{ABSTRACT.}

The Solid wastes has become in a major global problem because the vast majority of these are not being recovered, ending in landfills, open dumps or another illegal activities; A particular case are happening with the alcohol bottles made of glass, which have very little value in the traditional recycling economy but a high price in smuggle market. This research was made it in Neiva city in 2017 and determined the parameters of glass bottle waste management in night establishments in the urban area to identify the behaviour of the waste and be able to propose viable alternatives that will allow to be reincorporated into the cycle of lifetime.

For the procedure it was selected more than 20 degrees alcolimetric beverage bottles as indicator, work was done in the communes 3,4,6, because the largest population of establishments are located there. It was used the method of direct observation in each establishment and it were interviewed recycling entities and waste recovery cooperatives. It found the environmental management problems by the establishments a black market that arises from some informal recuperators and a product such as glass that is not considered economically viable within the local recovery process.

Keywords: Behavior, Management, Bottles, Glass, Establishments, Recilers.

\section{INTRODUCCIÓN.}

En Colombia 1 de cada 4 botellas de alcohol comercializadas es falsificada o adulterada, al punto que en el 2011 las incautaciones totales superaron las 700 mil botellas, una cifra superior en un $200 \%$ a las incautaciones cinco años atrás" (Zapata Giraldo et al., 2012), convirtiéndose en una problemática sanitaria y ambiental a nivel nacional(Monteiro, 2013).

Hoy dia la generación de las botellas de vidrio de bebidas alcohólicas se caracteriza por el bajo aprovechamiento dentro del reciclaje(Mata \& GÁLVEZ, 2014) y por el manejo indiscriminado que estas suelen tener en la etapa final del ciclo de vida, ya que es poco rentable su recuperación económicamente, puesto que el producto ocupa bastante volumen y pesa poco, convirtiéndose en un elemento que termina siendo depositado en un relleno sanitario(Victoria Calambas, Marmolejo Rebellón, \& Torres 
Lozada, 2012) o reincorporadose ilístamente del ciclo del alcohol adulterado generando riesgos para la salud humana.

Este estudio se realizóen el municipio de Neiva, capital del Departamento del Huila, ciudad intermedia con aproximadamente 388.000 habitantes (DANE, 2018), dueña de su propia marca de alcohol, presenta constantemente problemas por la adulteración y el contrabando de licores, encontrándose que a 2009 cerca de 37.690 botellas de 750 cc han sido adulteradas e incautadas por la DIJIN (Zapata \& Sabogal, 2012).

Esta investigación realizada en el municipio de Neiva determinó los parámetros de manejo y disposición de las botellas de vidrio de bebidas alcohólicas mayor a 20 grados, con el propósito de analizar el estado actual de este residuo y poder partir a generar investigaciones que permitan analizar el ciclo de vida y su reincorporación bajo el concepto de economía circular.

\section{METODOLOGÍA.}

La identificación de la generación de las botellas de vidrio de bebidas alcohólicas en los establecimientos nocturnos del municipio de Neiva tuvo como objetivo, determinar el ciclo de vida de las botellas de vidrio dentro de la ciudad, mediante observaciones directas a los establecimientos nocturnos, para conocer el manejo de las botellas de vidrio, para ello se realizó un recorrido por las comunas 3,4 y 6 de la ciudad de Neiva ubicando los diferentes establecimientos nocturnos dedicadas al comercio de bebidas alcohólicas, se levantó la información georeferenciada y se cruzó con las bases de datos con información oficial suministrada por Camara de Comercio de Neiva; Se diseñó una herramienta de observación directa para visualizar las condiciones de manejo de las botellas de vidrio, se identificaron comportamientos antes, durante y después del recorrido de la recolección de los residuos solidos por parte de la empresa prestadora de servicios. Se aplicó una muestra dirigida o intencional en dos poblaciones diferentes, en los establecimientos nocturno y cooperativas de reciclaje en la ciudad y se diseño y realizó una entrevista simple aplicada a 8 empresas recicladoras y cooperativas de reciclaje con el método dirigido o intencional.

\section{RESULTADOS.}

Según información suministrada por la Cámara de Comercio de Neiva 2017 hay cerca de 192 establecimientos nocturnos en la cuidad, los cuales 121 están repartidos entre las comunas 3,4,6, representando la población objetivo a valorar. Dentro de las características encontradas se clasificó 
por rango funcional cualitativo, agrupando tres categorías a trabajar, Disco Club, Bar Club, Bar Tienda Estanco.

De los recorridos se identificaron ciertos elementos que permiten analizar que las botellas de vidrio de bebidas alcohólicas son un material susceptible a ser despojado como elemento sin utilidad ya que dentro del mercado nacional su comercialización ha tendido a bajar por los bajos precios a nivel nacional (Consultores Limitada, 2011); Dentro de la información recolectada se pudo determinar que los establecimientos nocturnos no tienen un control adecuado sobre las botellas de vidrio de bebidas alcohólicas, ya que se verificó que son depositadas como cualquier otro elemento residual en las rutas previas de la Empresa Prestadora de Servicio de la ciudad(E.S.P.), por ello, su uso no está valorado como objeto que se reincorpore dentro del ciclo de vida.

Se encontró que el rol de recuperadores informales es fundamental dentro de la recuperación del material (Wilson, Velis, \& Cheeseman, 2006), ya que pueden operar sin control y bajo pésimas condiciones laborales (Viljoen, Blaauw, \& Schenck, 2016) y estar expuestos a diferentes tipos de riesgos por la manipulación de los residuos (Ballesteros, Yicenia, Urrego, Botero Botero, \& Arango, 2008), ya que su función es intervenir los residuos utilizando una técnica que consiste en levantar el contenido y realizar un movimiento pendular y un desplazamiento ascendente y descendente para identificar a través del sonido las botellas de vidrio de bebidas alcohólicas, realizando la selección, acopio y traslado de éstas.

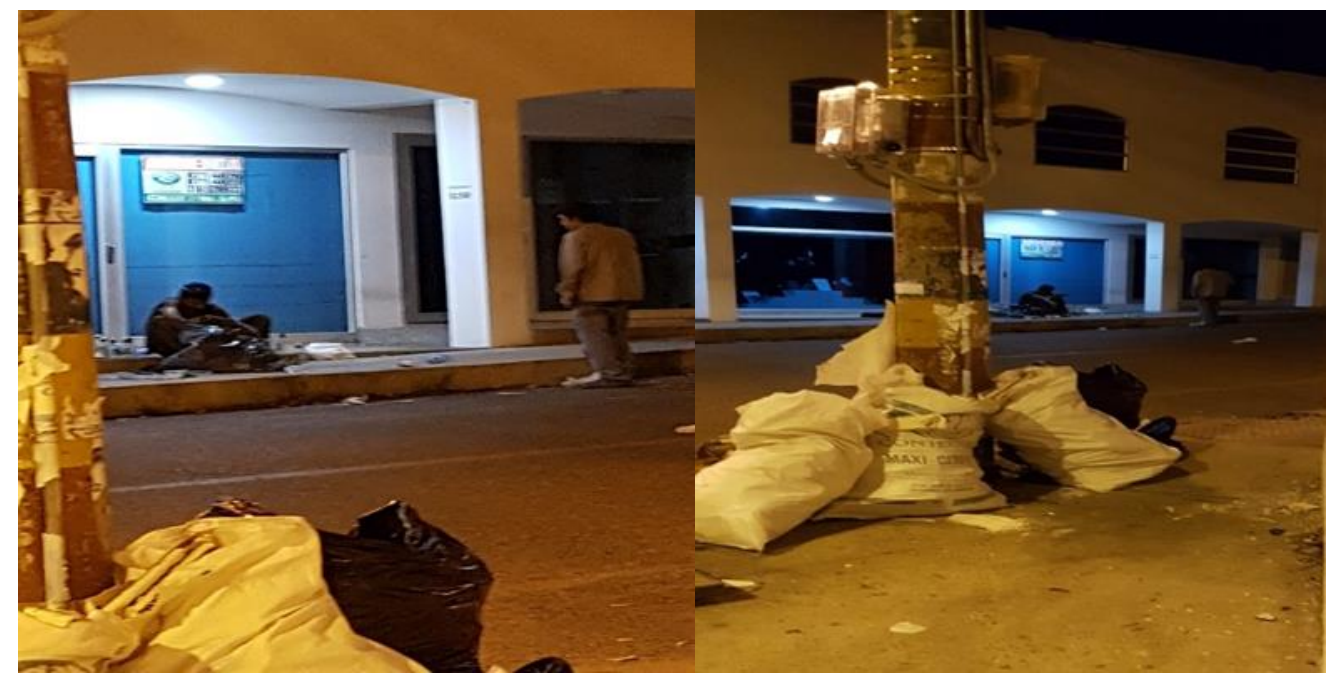

Figura 1. Desechos de establecimientos nocturnos, Municipio de Neiva

Por otro lado, se realizó el seguimiento a las recicladoras y cooperativas de reciclaje en jornadas de trabajo, donde se logró el acercamiento a 8 
de estas entidades dedicadas al comercio de material reciclable, se determinó que se encuentran legalmente constituidas el $87 \%$ de estas empresas recicladoras entrevistadas, datos altos comparados con la ciudad de Bogotá (Corredor, 2010), ya que la formalidad permite organizar y proponer estrategias de mejora de forma articulada(Saraiva de Souza, Bastos de Paula, \& de Souza-Pinto, 2012).

Se registró que el $100 \%$ de estas empresas comercializan los mismos productos recuperables, tales como el cartón, papel, plástico y chatarra, siendo un punto de partida para la gestión ambiental en esta cadena de comercialización (Durand, 2011); Se logró determinar que el 100\% de estas entidades no realizan la recuperación y comercialización de vidrio, donde el $75 \%$ de las recuperadoras mencionaron que la comercialización de este mercado es complejo y poco rentable(Consultores Limitada, 2011 ), el otro $25 \%$ de éstas han trabajado en la recuperación del material solo en ocasiones especiales utilizando el tema de protección de marca recibiendo apoyo de Secretaria de hacienda departamental y la DIAN.

\section{CONCLUSIONES.}

Teniendo en cuenta las situaciones de observación en campo en los establecimientos nocturnos, se concluyó que las botellas de vidrio de bebidas alcohólicas no tienen un control en su manejo, son elementos que se depositan como cualquier otro residuo, esta situación genera que su recolección este mediada por factores externos que intervienen en sus condiciones de acopio. Los recicladores informales son un pilar que Ocondicionan su trazabilidad una vez estas botellas de vidrio son dispuestas como residuos, estos factores son determinantes para comprender en su contexto como las botellas de vidrio son un material de fácil acceso como materia prima de vital importancia en la producción de licor adulterado, esto se dignifica en un mercado que emerge de la autosuficiencia de botellas de vidrio de bebidas alcohólicas que se reciclan, se distribuyen y comercializan de manera ilegal. Estas consideraciones tienen su explicación por la falta de articulación normativa específica para las botellas de vidrio de bebidas alcohólicas, la carencia de barreras por parte de las autoridades institucionales en la adquisición de estos elementos, y la inexistencia de un proceso que permita dar mayor incentivo a los establecimientos para el manejo de estas botellas de vidrio para su acopio. 


\section{BIBLIOGRAFÍA.}

Ballesteros, V. L., Yicenia, i, Urrego, C., Botero Botero, S., \& Arango, Y. L. (2008). Factores de riesgo biológicos en recicladores informales de la ciudad de Medellín, 2005 Biological risk factors in informal recyclers of Medellin city, 2005. Rev Fac Nac Salud Pública, 26(2), 177. https://doi.org/10.17533/udea.rfnsp

Consultores Limitada, A. (2011). Estudio Nacional del Reciclaje y los Recicladores Aproximación al Mercado de Reciclables y las Experiencias Significativas, 1, 53. Retrieved from http://cempre.org.co/wp-content/uploads/2017/05/3926estudio_nacional_de_reciclaje_aproximacicn_al_mercado_de_recicla bles_y_las_experiencias_significativas_0-1.pdf

Corredor, M. (2010). El sector reciclaje en Bogotá y su Región: Oportunidades para los negocios Inclusivos. Fundes, 60. Retrieved from http://asociacionrecicladoresbogota.org/wpcontent/uploads/2012/04/El_sector_reciclaje_en_Bogota_y.pdf

DANE. (2018). Información técnica Población 2018. Retrieved March 13, 2019, from https://www.dane.gov.co/index.php/estadisticas-portema/demografia-y-poblacion/censo-nacional-de-poblacion-yvivenda-2018/informacion-tecnica\#doc-ax

Durand, M. (2011). LA GESTIÓN DE LOS RESIDUOS SÓLIDOS EN LOS PAIISES EN DESARROLLO: ¿CÓMO OBTENER BENEFICIOS DE LAS DIFICULTADES ACTUALES? Solid Waste Management in the Less Developed Countries: How to Overcome Present Difficulties and to Obtain Benefits?, (23), 115-130. Retrieved from http://bibliotecavirtual.unad.edu.co/login?url=http://search.ebscoho st. com/login .aspx? direct $=$ true \&db=a9h\&AN=102936298\&lang=es\&s ite $=$ ehost-live

Mata, A., \& GÁLVEZ, I. N. G. C. (2014). Reciclaje de vidrio. Obtenido de Http://Genesis. Uag. Mx/Posgrado/Revistaelect/Calidad/Cal010. Pdf.

Monteiro, M. G. (2013). Alcohol y Salud Pública en América Latina: ¿cómo impedir un desastre sanitario? Adicciones (Vol. 25). Bogota: OPS. Retrieved from https://www.redalyc.org/html/2891/289126458003/

Saraiva de Souza, M. T., Bastos de Paula, M., \& de Souza-Pinto, H. (2012). O PAPEL DAS COOPERATIVAS DE RECICLAGEM NOS CANAIS REVERSOS PÓS-CONSUMO. THE ROLE OF RECYCLING COOPERATIVES IN THE REVERSE CHANNEL FOR POST- CONSUMER 
RECYCLABLES., 52(2), 246-262. Retrieved from http://bibliotecavirtual.unad.edu.co/login?url=http://search.ebscoho st.com/login .aspx ?direct $=$ true $\& d b=a 9 h \& A N=74732790 \&$ lang=es\&sit $\mathrm{e}=$ ehost-live

Victoria Calambas, F. A., Marmolejo Rebellón, L. F., \& Torres Lozada, P. (2012). ALTERNATIVAS PARA FORTALECER LA VALORIZACIÓN DE MATERIALES RECICLABLES EN PLANTAS DE MANEJO DE RESIDUOS SÓLIDOS EN PEQUEÑOS MUNICIPIOS. Ciencia e Ingeniería Neogranadina, 22(1), 59-73. Retrieved from http://www.redalyc.org/articulo.oa?id=91125275004\%0D

Viljoen, K., Blaauw, P., \& Schenck, R. (2016). "I would rather have a decent job": Potential barriers preventing street-waste pickers from improving their socio-economic conditions. South African Journal of Economic and Management Sciences VO - 19, (2), 175. https://doi.org/10.17159/2222-3436/2016/v19n2a2

Wilson, D. C., Velis, C., \& Cheeseman, C. (2006). Role of informal sector recycling in waste management in developing countries. Habitat International, 30(4), 797-808. https://doi.org/10.1016/J.HABITATINT.2005.09.005

Zapata Giraldo, J. G., Sabogal Moreno, A., Montes Viñas, A. C., Rodríguez, G., Castillo, J., \& contacto@fedesarrollo.org.co. (2012). Una estimación de la adulteración y la falsificación de bebidas alcohólicas en Colombia. SabMiller Bavaria. Retrieved from https://www.repository.fedesarrollo.org.co/handle/11445/2908

Zapata, J. G., \& Sabogal, A. (2012). Análisis del funcionamiento del monopolio de los licores en el Huila y diseño de una propuesta para mejorar su gestión. Retrieved from https://www.repository.fedesarrollo.org.co/handle/11445/320 